\title{
Evaluación espacial y temporal de la diversidad de los ascomicetes dulceacuícolas del canal turístico Santa Cruz, Xochimilco, México
}

\author{
Spatial and temporal evaluation of the diversity of freshwater Ascomycetes from the touristic \\ channel Santa Cruz, Xochimilco, Mexico
}

\author{
Allan Chavarria ${ }^{1 *}$, María C. González ${ }^{1}$ Edgar Dantán ${ }^{2}$ y Joaquín Cifuentes ${ }^{3}$ \\ ${ }^{1}$ Departamento de Botánica, Instituto de Biología, Universidad Nacional Autónoma de México. 04510 México D. F., México. \\ ${ }^{2}$ Centro de Investigación en Biotecnología, Universidad Autónoma del Estado de Morelos. 1001 Cuernavaca, Morelos, México. \\ ${ }^{3}$ Herbario FCME, Departamento de Biología, Facultad de Ciencias, Universidad Nacional Autónoma de México. 04510 México, D. F., México. \\ *Correspondencia: acchs2002@yahoo.com.mx
}

\begin{abstract}
Resumen. Se investigó la diversidad de los ascomicetes del canal Santa Cruz, considerado el de mayor importancia turística en Xochimilco, Distrito Federal. En 2007 se realizaron 2 muestreos en las épocas de menor y mayor precipitación. En el márgen del canal se establecieron 4 estaciones de muestreo con diferente grado de urbanización y en cada una se sumergieron bloques de madera de Virola sp., Tabebuia donnell-smithii, Bucida buceras, Pinus sp., Cupressus lindlei y Abies religiosa durante 8 semanas. Se midió la temperatura, el pH, el oxígeno disuelto, la materia orgánica y el fósforo, y se determinó el número más probable de bacterias coliformes del agua. Para evaluar los datos, se aplicaron el índice de diversidad de Shannon y el índice de similitud de Sørensen. La mayor diversidad de ascomicetes $\left(H^{\prime}=1.152\right)$ se registró en la tercera estación, ubicada en la zona de viveros con flores y la menor diversidad $\left(H^{\prime}=0.976\right)$ se obtuvo en la primera estación, localizada frente al embarcadero Santa Cruz, ambos valores de diversidad coincidieron con el grado de urbanización y con los datos fisicoquímicos y bacteriológicos. La temporada de lluvias presentó la diversidad más baja debido probablemente al efecto de la precipitación en este ambiente acuático.
\end{abstract}

Palabras clave: ambiente léntico, bacterias coliformes, Ascomycetes dulceacuícolas, madera en sumersión, micología, taxonomía.

\begin{abstract}
The diversity of freshwater Ascomycetes was determined in the touristic channel Santa Cruz, Xochimilco, Mexico. Two surveys were conducted in 2007 during the dry and wet season. Four sampling sites were selected in the channel margin, based on the level of urbanization. At each sampling site, wood panels of Tabebuia donnell-smithii, Bucida buceras, Pinus sp., Cupressus lindlei and Abies religiosa were submerged during 8 weeks. Water temperature, dissolved oxygen, organic matter, phosphorus and most probable number of coliform bacteria were determined. Results were evaluated using the diversity index of Shannon and the index of similarity of Sørensen. The highest Ascomycete diversity recorded $\left(H^{\prime}=1.152\right)$ was found at the third sampling site, located near commercial florist greenhouses. The lowest diversity $\left(H^{\prime}=0.976\right)$ was obtained at the first sampling site, in front of Santa Cruz pier. Both values of diversity were in agreement with the urbanization level and physicochemical and bacteriological data. The wet season had the lowest diversity of freshwater Ascomycetes, perhaps due to the effect of the rainfall in this aquatic environment.
\end{abstract}

Key words: lentic environment, coliform bacteria, freshwater Ascomycetes, submerged wood mycology, taxonomy.

\section{Introducción}

Los ascomicetes dulceacuícolas son los que completan su ciclo de vida total o parcialmente en diversos ambientes acuáticos, lénticos y lóticos, donde su principal función ecológica es degradar los restos orgánicos, contribuyendo en esta forma al reciclaje de los nutrimentos (Goh y Hyde, 1996; Wong et al., 1998; Tsui et al., 2003).

Recibido: 03 julio 2009; aceptado: 15 enero 2010
Los estudios sobre la diversidad de los Ascomycetes que se desarrollan en la madera sumergida de los cuerpos de agua naturales o perturbados, tanto de zonas tropicales como templadas, son escasos y recientes (Hyde y Goh, 1998; Garnett et al., 2000; Ho et al., 2002; Pascoal et al., 2005; Cai et al., 2006). La mayoría de las especies que se han registrado son cosmopolitas, aunque algunas pueden ser más comunes en las regiones templadas que en las tropicales, mientras que otras pueden tener una distribución restringida en zonas geográficas específicas 
(Wong et al., 1998).

Poco se conoce de la diversidad fúngica que habita en los ambientes acuáticos del mundo. . De las 97330 especies de hongos descritas, sólo se han registrado de ambientes acuáticos 3 047; de éstas, 1384 especies son de agua dulce con 1145 ascomicetes (Hawksworth, 2001; Hyde, 2001; Shearer et al., 2007; Kirk et al., 2008). En México, solamente están registradas 8 de las 1145 especies de ascomicetes dulceacuícolas sensu stricto; 3 fueron registradas en los canales de Xochimilco, Distrito Federal (González y Chavarría, 2005), 2 en Los Tuxtlas, Veracruz (Castañeda et al., 2004, 2005) y 3 en la Laguna de las Ilusiones, Tabasco (Rosique et al., 2008).

En la zona hidrológica de Xochimilco que se localiza al sur de la zona metropolitana de la ciudad de México hay $189 \mathrm{~km}$ de canales que han sido contaminados por el aporte de aguas residuales, desechos domésticos, industriales, productos agrícolas y filtraciones del sistema de drenaje. Lo anterior ha afectado diferentes especies de seres vivos que habitan en la zona, provocando una disminución en su biodiversidad (Juárez-Figueroa et al., 2003).

De todos los canales que forman la red hidrológica de Xochimilco, el canal turístico Santa Cruz es el de mayor importancia turística y cultural, y uno de los más frecuentados durante todo el año por visitantes locales, nacionales y extranjeros. A pesar de su importancia, se desconoce la diversidad de los ascomicetes dulceacuícolas que habitan en los restos de madera sumergida, por lo que el objetivo de este trabajo fue recolectar, describir y determinar la diversidad biológica de los Ascomycetes dulceacuícolas presentes en 4 sitios del canal Santa Cruz, Xochimilco durante la temporada seca y lluvias.

\section{Materiales y métodos}

Área de estudio. El canal turístico Santa Cruz se encuentra en la zona del Embarcadero de Nativitas, en la Delegación Xochimilco, Distrito Federal, tiene cerca de 2 kilómetros de longitud, una profundidad que va de 50 a 170 centímetros y.está a una altitud aproximada de 240 metros. En sus márgenes se encuentran invernaderos donde se cultivan flores y plantas ornamentales, pequeñas zonas agrícolas y casas habitación con diverso grado de desarrollo urbano (Figs. 1 y 2). La zona presenta un clima tropical de altitud elevada, con una temperatura media anual de $16{ }^{\circ} \mathrm{C}$ la mayor parte del año. La época seca es de enero a mayo y la de lluvias de junio a septiembre (Zambrano et al., 2009; CONAGUA, 2009).

Muestreo. En el canal turístico Santa Cruz, se establecieron 4 estaciones de muestreo, cada una con diferente grado de actividad antropogénica en sus márgenes. Se realizaron 2 muestreos, uno en febrero y otro en agosto de 2007, meses en los que se registraron la mínima y la máxima precipitación de la temporada seca y lluvias, respectivamente.

En ambos muestreos se siguió la metodología propuesta por Jones (1971). con la modificación que más adelante se indica en este mismo párrafo. En cada lugar de muestreo se sumergieron 3 de las así llamadas carnadas que consisten en 6 bloques de madera rectangulares de $10 \times 20 \times 2 \mathrm{~cm}$. En cada bloque se practicaron 2 perforaciones a $5 \mathrm{~cm}$ de cada extremo. Los 6 bloques de madera de cada carnada se separaron con segmentos de $2 \mathrm{~cm}$ de largo de manguera de hule, ( $2 \mathrm{~cm}$ de diámetro), y se alinearon a la altura de las perforaciones. Los 6 bloques se unieron pasando por los orificios una cuerda de nylon. Al extremo inferior de la carnada se amarró un lastre para mantenerla sumergida, mientras que la cuerda del extremo superior sirvió para sujetarla a la orilla del canal. Con el fin de obtener una mayor diversidad de hongos, se emplearon 6 bloques de diferentes maderas: Virola sp., Tabebuia donnell-smithii, Bucida buceras, Pinus sp., Cupressus lindlei y Abies religiosa, en lugar de 2 bloques de un solo tipo de madera (Pinus sp.) como propone el método de Jones (1971). Las carnadas se dejaron sumergidas durante 8 semanas, al término de las cuales se sacaron del agua y se transportaron al laboratorio donde se procesaron antes de 24 horas.

Procesamiento de las muestras. Los bloques de madera de cada carnada fueron separados y se colocaron individualmente en una cámara húmeda, que consiste en una caja de plástico estéril con tapa hermética a la que previamente se le colocaron en el fondo servilletas de papel estériles y humedecidas con agua destilada estéril. Las cámaras se incubaron durante 4 semanas a la temperatura del laboratorio $\left(25-30{ }^{\circ} \mathrm{C}\right)$, expuestas a una iluminación diaria con $12 \mathrm{~h}$ de luz natural y $12 \mathrm{~h}$ de oscuridad. Al concluir el periodo de incubación, se examinó la superficie de los bloques para localizar las estructuras reproductoras de los ascomicetes. Se identificó cada ascomicete presente en cada bloque y se preservó deshidratándolo sobre el sustrato y en preparaciones microscópicas permanentes. Cuando fue posible, los hongos se cultivaron hasta obtener cultivos puros utilizando agar jugo V-8 (agar 20 g, carbonato de calcio 3 g, jugo V8 Campbell® $180 \mathrm{ml}$, agua destilada 1 litro) y agar harina de maíz (agar 17 g BD BBL®, agua destilada 1 litro) (Ho et al., 1997; Choi et al., 1999; Cai et al., 2003; Goh y Tsui, 2003).

Los ejemplares preservados se depositaron en la Colección de Hongos del Herbario Nacional (MEXU). En cada lugar de muestreo se determinó la ubicación geográfica exacta y se tomaron otros datos de colecta in situ como el $\mathrm{pH}$, oxígeno disuelto y temperatura del agua (oxímetro portátil, model 51B, YSI, Inc., Ohio). En el laboratorio, se determinó la materia orgánica disuelta 

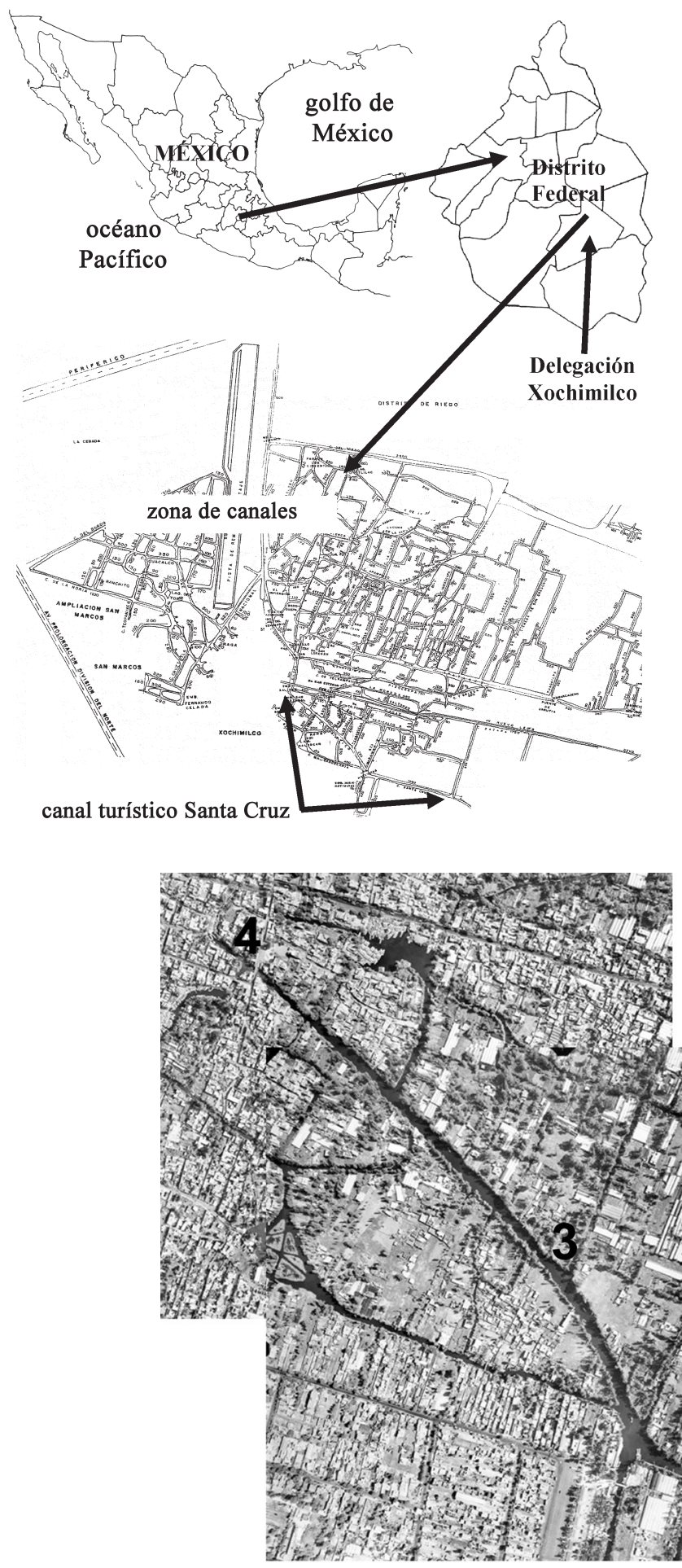

Figura 2. Fotografía aérea del canal turístico Santa Cruz, Xochimilco, donde se indica la localización de las 4 estaciones de muestreo.
Figura 1. Ubicación del canal turístico Santa Cruz en la zona de canales de Xochimilco, México.

mediante la demanda química de oxígeno por la técnica de reflujo, el fósforo soluble por la técnica del ácido ascórbico, y el número más probable de bacterias coliformes totales y fecales mediante la técnica de fermentación de tubos múltiples (APHA, 1992).

Análisis de los datos. La diversidad de los ascomicetes que se registró en los 4 lugares de muestreo y durante las 2 épocas del año, se listó y acomodó en orden descendente según el valor de abundancia relativa (AR) de cada taxa. De esta manera, los valores ordenados de AR permitieron distinguir los grupos de ascomicetes dominantes (AR $\geq$ $5.0 \%)$, comunes (AR $\geq 1.0 \%)$ y $\operatorname{raros}(\mathrm{AR}<1 \%)(\mathrm{Ho}$ et al., 2002). El porcentaje de abundancia es el número de frecuencias de aparición de un taxa (n) dividida entre el número total de frecuencias recuperadas de la muestra (Bills y Polishook, 1994).

Para evaluar la diversidad espacial y temporal de los ascomicetes del canal turístico Santa Cruz, los datos se analizaron aplicando índices de estadística ecológica de similitud y diversidad. La similitud entre la composición de los ascomicetes de las comunidades estudiadas se analizó aplicando el índice de similitud $S=2 C / a+b \times 100$, donde: $S=$ índice de similitud, $C=$ número de especies comunes para ambos puntos de muestreo, $a=$.número de especies del primer punto de muestreo y $b=$ número de especies 
del segundo muestreo (Sørensen, 1948). Este índice se expresa como valores que van de 0 a 100, para cuantificar desde la disimilitud total hasta la semejanza completa, respectivamente. La diversidad de los ascomicetes se evaluó mediante el índice de Shannon expresado como:

$$
H^{\prime}=-\sum_{i=1}^{S p i} \ln p i
$$

donde: $H^{\prime}=$ diversidad de individuos, $S=$ número de especies, $p i=$ proporción del número de individuos de la especie $i$ con respecto al total (Moreno, 2001).

\section{Resultados}

Como resultado general de los 2 muestreos que se realizaron en el canal turístico Santa Cruz se obtuvieron 28 Ascomycetes (13 meiospóricos y 15 mitospóricos) pertenecientes a la clase Sordariomycetes en los órdenes Sordariales, Microascales, Hypocreales y Diaporthales; a la clase Dothidemycetes en los órdenes Pleosporales y Capnodiales, y a la clase Orbiliomycetes en el orden Orbiliales (Figs. 3-12).

En relación con su grupoecológico, la diversidadfúngica que se registró en el canal turístico Santa Cruz incluyó 9 ascomicetes dulceacuícolas estrictos, 9 ascomicetes dulceacuícolas facultativos y 6 ascomicetes terrestres. Ascolacicola aquatica, Naïs inornata y Triadelphia uniseptata se registran por primera vez en Xochimilco. Los ascomicetes más abundantes fueron Passalora clematidis (18\%), Arthrobotrys oligospora (14.4\%), Phomopsis sp. (13.3\%) y Graphium sp. (11.7\%), los cuales se encontraron en las 4 estaciones y en la temporada seca y de lluvias, además, se trata de ascomicetes mitospóricos considerados ecológicamente como facultativos y terrestres (Cuadro 1).

Los resultados de la evaluación espacial mostraron que la segunda estación de muestreo presentó el mayor porcentaje de abundancia, mientras que en la tercera estación se registró la mayor diversidad de ascomicetes $\left(H^{\prime}=1.152\right)$ (Cuadro 1). En la segunda estación y en la tercera se presentaron la mayor abundancia y diversidad de ascomicetes dulceacuícolas estrictos. Passalora clematidis fue la especie más abundante en las estaciones de muestreo primera, tercera y cuarta (Cuadro 1). Los resultados del análisis de similitud muestran que la diversidad de los ascomicetes fue similar en las 4 estaciones, y las que mayor similitud tuvieron entre sí fueron la segunda y tercera $(S=$ $82.5 \%)$.

Los resultados del análisis temporal indican que en la temporada seca se registró el número de frecuencias más elevado y la mayor diversidad de ascomicetes $\left(H^{\prime}=\right.$ 1.144) (Cuadro 2). Passalora clematidis fue la especie más abundante en la temporada seca, y $A$. oligospora en la de lluvias. Los ascomicetes menos abundantes, como Dictyosporium sp., Lasiosphaeria sp., Nectria rishbethii, Ophioceras commune y Ceratocystis sp., se registraron sólo una vez. La similitud entre la composición de los ascomicetes registrados en la temporada seca y la de lluvias fue alta $(S=66.6 \%)$. Arthrobotrys oligospora, $A$. aquatica, Graphium sp., N. inornata, P. guttulata, Phoma sp., Phomopsis sp., Podospora sp., Taeniolella rudis, Trichocladium sp. y T. uniseptata se registraron tanto en la temporada seca como en la de lluvias, predominando los grupos terrestres y facultativos, así como las formas mitospóricas.

\section{Discusión}

Ciertas líneas evolutivas de los ascomicetes han tenido más éxito que otras para adaptarse al ambiente acuático de agua dulce El orden Sordariales es el que incluye más especies (114 spp.) (Shearer et al., 2006), lo que está de acuerdo con los resultados de este trabajo, ya que fue el que presentó el mayor número de especies (6 spp.). En general, la diversidad de los ascomicetes que se registró en este trabajo es similar a la de los cuerpos de agua urbanos, tanto de México como de otros países (Cuadro 3). Al comparar la diversidad de los ascomicetes dulceacuícolas que se obtuvo en este trabajo $\left(H^{\prime}=1.009\right)$ con los valores que se registraron en 2 estudios realizados en la misma área de estudio, la misma época del año (temporada de lluvias) y con la misma metodología en los años 2003 ( $H^{\prime}=$ $1.258)$ y $2005\left(H^{\prime}=1.158\right)$ se observa que la diversidad de los ascomicetes ha bajado gradualmente, debido probablemente al efecto de las actividades antropogénicas y/o al cambio climático, resultado del desarrollo urbano de la ciudad de México (Chavarria, 2003, 2005) (Cuadro 3). Al comparar la diversidad de los ascomicetes que se encontró en este estudio con los resultados registrados en otros cuerpos de agua tropicales y subtropicales ubicados en zonas urbanas, como Laguna de las Ilusiones, Tabasco, México, y el lago Dianchi, Yunnan, China, la diversidad que se registró en el canal Santa Cruz es mayor, por lo que probablemente se trata de una zona con alta diversidad (Chavarria, 2003, 2005; Lou et al., 2004; Rosique, 2008) (Cuadro 3). Sin embargo, en el canal turístico Santa Cruz los ascomicetes con los mayores valores de abundancia fueron los facultativos y los terrestres (Cuadro 1), resultado que podría estar indicando una pérdida de la diversidad propia del ecosistema acuático que está representada por los ascomicetes considerados estrictos, que son los que 

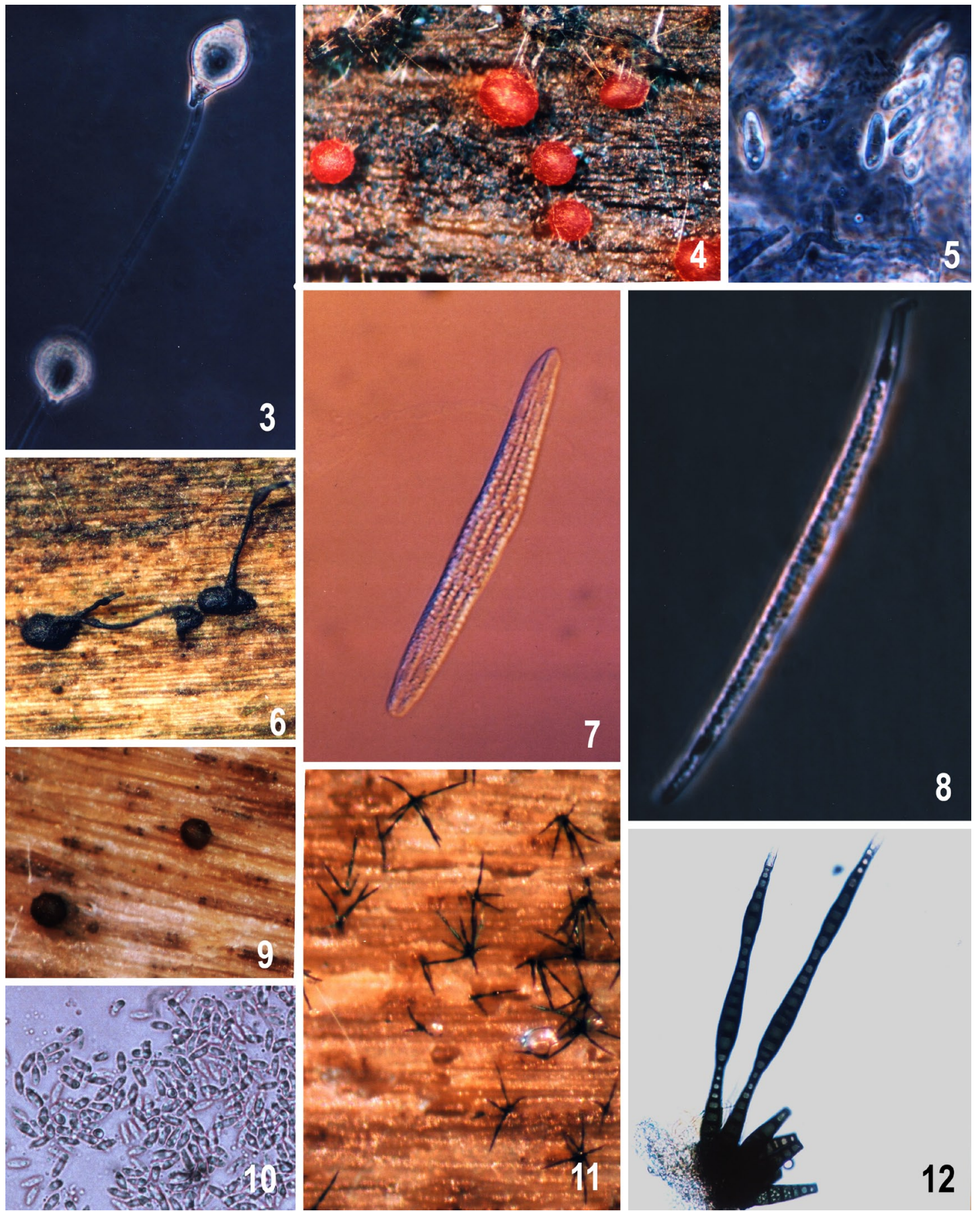

Figuras 3-12. Ascomicetes dulceacuícolas lignícolas del canal turístico Santa Cruz, Xochimilco. 3. Conidiosporas de Monotospora, $\times$ 1110. 4. Ascomas rojos, globosos de Nectria rishbethii, $\times 60$. 5. Ascosporas hialinas de Nectria rishbethii, $\times 1000.6$. Ascomas oscuros, globosos, con largos cuellos (flecha), semi-inmersos en la madera, de Ophioceras commune, $\times 30$. 7. Asca cilíndrica con ascosporas de Ophioceras commune, $\times 250$. 8. Ascospora filiforme, hialina, unicelular de Ophioceras commune, $\times 1000.9$. Picnidios marrones globosos sobre madera, de Phomopsis sp., $\times$ 50. 10. Conidiosporas hialinas unicelulares de Phomopsis sp. $\times 1100$. 11. Grupos de conidióforos sobre la madera de Taeniolella rudis, $\times 60$. 12. Conidióforo de Taeniolella rudis, $\times 400$. Fotografías $3,5,8$ y 10 tomadas con microscopía de contraste de fases y fotografía 7 con microscopía de contraste diferencial de Nomarski. 
Cuadro 1. Abundancia y diversidad de los ascomicetes registrados en las 4 estaciones de muestreo en el canal Santa Cruz, Xochimilco, México, Distrito Federal

\begin{tabular}{|c|c|c|c|c|c|c|}
\hline \multirow[t]{2}{*}{ Ascomicetes } & \multicolumn{4}{|c|}{ Estaciones de muestreo } & \multirow{2}{*}{$\begin{array}{c}\text { Abundancia } \\
\text { (n) }\end{array}$} & \multirow{2}{*}{$\begin{array}{c}A R \\
\%\end{array}$} \\
\hline & 1 & 2 & 3 & 4 & & \\
\hline Passalora clematidis ${ }^{\mathrm{MI} * *}$ & 14 & 10 & 15 & 15 & 54 & 18.0 \\
\hline Arthrobotrys oligospora ${ }^{\mathrm{MI} * * *}$ & 13 & 11 & 10 & 9 & 43 & 14.4 \\
\hline Phoma sp. ${ }^{\mathrm{MI} * *}$ & 12 & 15 & 9 & 4 & 40 & 13.3 \\
\hline Graphium sp. ${ }^{\mathrm{MI} * * *}$ & 9 & 10 & 4 & 12 & 35 & 11.7 \\
\hline Phomopsis sp. ${ }^{\mathrm{MI} * *}$ & 8 & 8 & 2 & 3 & 21 & 7.0 \\
\hline Petriella guttulata $\mathrm{ME} * * * *$ & 6 & 5 & 2 & 3 & 16 & 5.3 \\
\hline Ascolacicola aquatica $^{\mathrm{ME} *}$ & 2 & 4 & 4 & 2 & 12 & 4.0 \\
\hline Podospora sp. ${ }^{\mathrm{ME} * * *}$ & 4 & 1 & 2 & 4 & 11 & 3.6 \\
\hline No identificado $1^{\mathrm{MI}}$ & 0 & 1 & 3 & 6 & 10 & 3.3 \\
\hline Bactrodesmium sp. ${ }^{\mathrm{MI} *}$ & 0 & 3 & 4 & 1 & 8 & 2.6 \\
\hline Monotosporella sp. ${ }^{\mathrm{MI} *}$ & 0 & 2 & 2 & 2 & 6 & 2.0 \\
\hline Nä̈s inornata $\mathrm{ME} *$ & 0 & 2 & 1 & 2 & 5 & 1.6 \\
\hline Taeniolella rudis ${ }^{\mathrm{MI} * *}$ & 1 & 2 & 2 & 0 & 5 & 1.6 \\
\hline Trichocladium sp. ${ }^{\mathrm{MI} *}$ & 2 & 1 & 1 & 1 & 5 & 1.6 \\
\hline 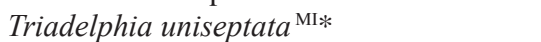 & 0 & 3 & 1 & 0 & 4 & 1.3 \\
\hline Bombardia sp. ${ }^{\mathrm{ME} *}$ & 0 & 3 & 0 & 0 & 3 & 1.0 \\
\hline Massariana sp. ${ }^{\mathrm{ME} *}$ & 1 & 0 & 1 & 1 & 3 & 1.0 \\
\hline No identificado $1^{\mathrm{ME}}$ & 0 & 3 & 0 & 0 & 3 & 1.0 \\
\hline Chaetomium globosum $^{\mathrm{ME} * *}$ & 0 & 1 & 1 & 0 & 2 & 0.6 \\
\hline Fusarium sp. ${ }^{\mathrm{MI} * * *}$ & 0 & 1 & 1 & 0 & 2 & 0.6 \\
\hline Helicosporium sp. ${ }^{\mathrm{MI} * *}$ & 0 & 0 & 2 & 0 & 2 & 0.6 \\
\hline No identificado $2^{\text {MI }}$ & 2 & 0 & 0 & 0 & 2 & 0.6 \\
\hline No identificado $2^{\mathrm{ME}}$ & 2 & 0 & 0 & 0 & 2 & 0.6 \\
\hline Ceratocystis sp. ${ }^{\mathrm{ME} * * *}$ & 0 & 0 & 0 & 1 & 1 & 0.3 \\
\hline Lasiosphaeria sp. $^{\mathrm{ME} * *}$ & 0 & 0 & 1 & 0 & 1 & 0.3 \\
\hline Nectria rishbethii ${ }^{\mathrm{ME} * *}$ & 0 & 0 & 1 & 0 & 1 & 0.3 \\
\hline Dictyosporium sp. ${ }^{\mathrm{MI} * *}$ & 0 & 0 & 0 & 1 & 1 & 0.3 \\
\hline Ophioceras commune $e^{\mathrm{ME} *}$ & 0 & 0 & 1 & 0 & 1 & 0.3 \\
\hline Frecuencias totales & 76 & 86 & 70 & 67 & 299 & \\
\hline$\%$ de frecuencias & 25.4 & 28.7 & 23.4 & 22.4 & & 100 \\
\hline Número total de ascomicetes & & & & & & \\
\hline Número total de ascomicetes por estación & 13 & 19 & 22 & 16 & & \\
\hline Índice de diversidad $\left({ }^{\prime} \mathrm{H}\right)$ & 0.976 & 1.123 & 1.152 & 1.030 & & \\
\hline Equidad ('J) & 0.876 & 0.878 & 0.858 & 0.855 & & \\
\hline
\end{tabular}

MIascomicete mitospórico, ${ }^{\mathrm{ME}}$ ascomicete meiospórico; *ascomicete estricto, **ascomicete facultativo, ***ascomicete terrestre; $A R=$ abundancia relativa.

solamente se han registrado como habitantes de cuerpos de agua naturales, sin perturbación, y que presentan adaptaciones para poder crecer, reproducirse y dispersarse en dicho ambiente. Ante la posible pérdida de su diversidad, es probable que los ascomicetes dulceacuícolas estrictos en el canal turístico Santa Cruz estén siendo reemplazados por ascomicetes de origen terrestre, como los facultativos, que también pueden habitar en el medio acuático y completar su ciclo de vida en él, y los propiamente terrestres, que sólo habitan en dicho ambiente por corto tiempo y no pueden completar su ciclo de vida.
La diversidad de los ascomicetes registrada en otros cuerpos de agua naturales y sin perturbación, como en Yumká, Tabasco, México (Martínez, 2009) y en el lago Barrine, en Queensland, Australia, (Hyde y Goh, 1998), está representada por la alta abundancia y diversidad de los ascomicetes estrictos, por lo que su biodiversidad se encuentra bien conservada. Probablemente la abundancia baja de ascomicetes dulceacuícolas estrictos registrada en las 2 épocas del año se deba a que las características limnológicas del canal turístico Santa Cruz no son propicias para el desarrollo de estos organismos. Los ascomicetes 
Cuadro 2. Abundancia y diversidad de los ascomicetes registrados durante la temporada seca y de lluvias en el canal turístico Santa Cruz, Xochimilco, México, Distrito Federal

\begin{tabular}{|c|c|c|c|c|}
\hline Ascomicetes & $\begin{array}{l}\text { Temporada } \\
\text { seca }\end{array}$ & $\begin{array}{l}\text { Temporada } \\
\text { lluvias }\end{array}$ & $\begin{array}{c}\text { Abundancia } \\
\text { (n) }\end{array}$ & $\begin{array}{c}A R \\
\%\end{array}$ \\
\hline Passalora clematidis ${ }^{\mathrm{MI} * *}$ & 31 & 23 & 54 & 18.0 \\
\hline Arthrobotrys oligospora ${ }^{\mathrm{MI} * * *}$ & 16 & 27 & 43 & 14.4 \\
\hline Phoma sp. ${ }^{\mathrm{MI} * *}$ & 26 & 14 & 40 & 13.3 \\
\hline Graphium sp. ${ }^{\mathrm{MI} * * *}$ & 23 & 12 & 35 & 11.7 \\
\hline Phomopsis sp. ${ }^{\mathrm{MI} * *}$ & 11 & 10 & 21 & 7.0 \\
\hline Petriella guttulata $\mathrm{ME} * * *$ & 15 & 1 & 16 & 5.3 \\
\hline Ascolacicola aquatica $^{\mathrm{ME} *}$ & 1 & 11 & 12 & 4.0 \\
\hline Podospora sp. ${ }^{\mathrm{ME} * * *}$ & 9 & 2 & 11 & 3.6 \\
\hline No identificado $1^{\mathrm{MI}}$ & 9 & 1 & 10 & 3.3 \\
\hline Bactrodesmium sp. ${ }^{\mathrm{MI} *}$ & 0 & 8 & 8 & 2.6 \\
\hline Monotosporella sp. ${ }^{\mathrm{MI} *}$ & 6 & 0 & 6 & 2.0 \\
\hline Naïs inornata ${ }^{\mathrm{ME} *}$ & 2 & 3 & 5 & 1.6 \\
\hline Taeniolella rudis ${ }^{\mathrm{MI} * *}$ & 4 & 1 & 5 & 1.6 \\
\hline Trichocladium sp. ${ }^{\mathrm{M}} *$ & 3 & 2 & 5 & 1.6 \\
\hline Triadelphia uniseptata $\mathrm{MI} *$ & 2 & 2 & 4 & 1.3 \\
\hline Bombardia sp. ${ }^{\mathrm{ME} *}$ & 3 & 0 & 3 & 1.0 \\
\hline Massariana sp. ${ }^{\mathrm{ME} *}$ & 3 & 0 & 3 & 1.0 \\
\hline No identificado 1 ME** & 2 & 1 & 3 & 1.0 \\
\hline Chaetomium globosum ${ }^{\mathrm{ME} * *}$ & 2 & 0 & 2 & 0.6 \\
\hline Fusarium sp. ${ }^{\mathrm{MI} * * *}$ & 2 & 0 & 2 & 0.6 \\
\hline Helicosporium sp. ${ }^{\mathrm{MI} * *}$ & 2 & 0 & 2 & 0.6 \\
\hline No identificado $2^{\mathrm{MI}}$ & 2 & 0 & 2 & 0.6 \\
\hline No identificado $2^{\mathrm{ME}}$ & 0 & 2 & 2 & 0.6 \\
\hline Ceratocystis sp. ${ }^{\mathrm{ME} * * *}$ & 1 & 0 & 1 & 0.3 \\
\hline Lasiosphaeria sp. $^{\mathrm{ME} * *}$ & 0 & 1 & 1 & 0.3 \\
\hline Nectria rishbethii $\mathrm{ME} * *$ & 1 & 0 & 1 & 0.3 \\
\hline Dictyosporium sp. ${ }^{\mathrm{MI} * *}$ & 1 & 0 & 1 & 0.3 \\
\hline Ophioceras commune ME* $^{\mathrm{M}}$ & 0 & 1 & 1 & 0.3 \\
\hline Frecuencias totales & 177 & 122 & 299 & \\
\hline$\%$ de frecuencias & 59.2 & 40.8 & & 100 \\
\hline Número total de ascomicetes & & 28 & & \\
\hline Número total de ascomicetes por muestreo & 24 & 18 & & \\
\hline Índice de diversidad $\left({ }^{\prime} \mathrm{H}\right)$ & 1.144 & 1.009 & & \\
\hline Equidad ('J) & 0.829 & 0.804 & & \\
\hline
\end{tabular}

MIascomicete mitospórico, ${ }^{\mathrm{ME}}$ ascomicete meiospórico; *ascomicete estricto, **ascomicete facultativo, ***ascomicete terrestre; $A R=$ abundancia relativa.

dulceacuícolas estrictos que se encontraron en Xochimilco han sido registrados en ambientes tropicales y templados (Shearer y Crane, 1986; Sivichai et al., 2000; Ho et al., 2002).

La presencia de hongos terrestres en el agua está registrada en diversos trabajos (Shearer y Crane, 1986; Révay y Gönczöl, 1990; Au et al., 1992; Vukojevic et al., 1997; Hyde y Goh, 1998; Fryar et al., 2004; Cai et al., 2006). Los hongos terrestres presentes en los restos vegetales que ingresan al agua son desplazados de manera natural por especies dulceacuícolas con el paso del tiempo. Sin embargo, en ambientes contaminados con metales pesados,
DDT, fungicidas, herbicidas o contaminación orgánica, el desarrollo de muchas especies de hongos acuáticos puede inhibirse o afectarse alterando su diversidad; no obstante, este cambio no significa pérdida de la función ecológica de estos organismos (Krauss et al., 2003).

Passalora clematidis es una especie cosmopolita y saprobia registrada en diferentes sustratos vegetales en ambientes dulceacuícolas lénticos y lóticos de zonas templadas y tropicales (Shearer y Crane, 1986; Hyde y Goh, 1998; Sivichai et al., 2000; Cai et al., 2002; Ho et al., 2002; Lou et al., 2004). Este hongo se ha encontrado en ambientes dulceacuícolas contaminados con fertilizantes 


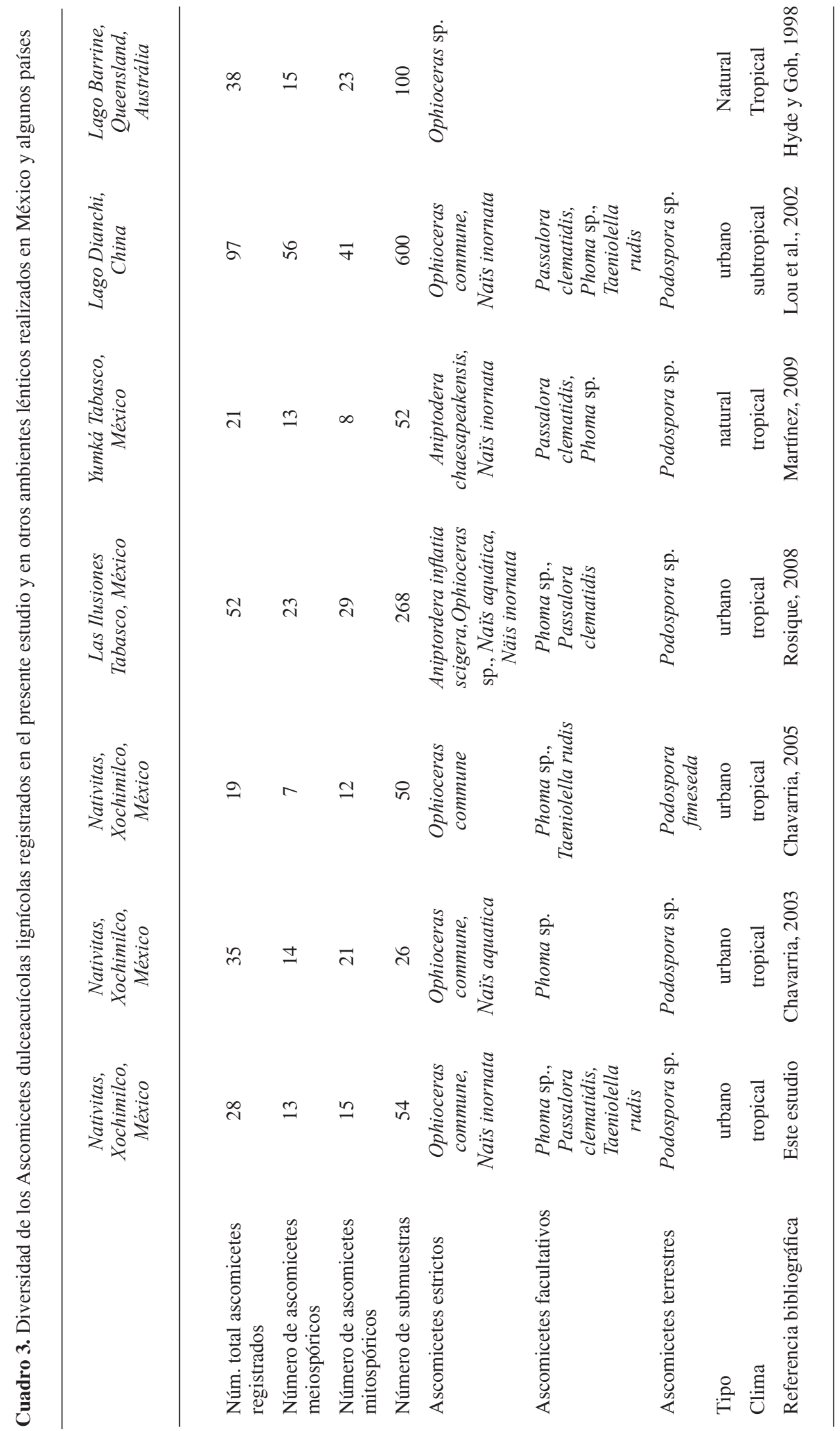


químicos o con aguas domésticas e industriales (Lou et al., 2004). En México se ha registrado en el estado de Veracruz, creciendo sobre restos vegetales presentes en el suelo, y en el estado de Tabasco en carnadas de madera sumergidas en la Laguna de las Ilusiones (Heredia et al., 1997; Rosique, 2008). Presenta conidióforos erectos sobre la madera que posiblemente le permiten una mejor dispersión de las esporas cuando el sustrato está parcialmente expuesto al aire (Hyde y Goh, 2003).

Arthrobotrys oligospora es un hongo del suelo que se ha registrado como saprobio de materia vegetal y en carnadas de madera enterradas en el suelo (Domsch et al., 1980). Hay registros ocasionales de este orgnismo en ambientes dulceacuícolas tropicales y templados (Kane et al., 2002; Fryar et al., 2004). Su teleomorfo es Orbilia auricolor, un discomicetes que crece sobre la madera (Pfister y Liftik, 1995). Algunas especies de Orbilia se han registrado esporádicamente en cuerpos de agua dulce.

Las especies del género Phoma poseen una amplia distribución geográfica y se pueden encontrar en numerosos nichos ecológicos como saprobios o parásitos de plantas, animales, hongos, oomycetes y seres humanos (Aveskamp et al., 2008). Se le ha registrado en China y Estados Unidos creciendo sobre madera en ambientes dulceacuícolas (Shearer y Crane, 1986; Cai et al., 2002; Ho et al., 2002). En México se registró una especie de Phoma en estanques de estabilización para tratar aguas residuales domésticas (Carranco et al., 1984).

Del género Nectria se han registrado especies en China y Hungría, creciendo sobre madera sumergida (Revay y Gönczöl, 1990; Luo et al., 2004). Uno de sus anamorfos se encuentra en el género Fusarium, el cual posee especies clasificadas como limafilas (Au et al., 2002).

Taeniolella rudis es una especie rara; se encuentra en Europa y América del Norte, tanto en ambientes terrestres como dulceacuícolas; en México, sólo en Xochimilco durante la temporada de lluvias.

Podospora sp. es un ascomicete que se asocia con la presencia de contaminación de origen orgánico (Luo et al., 2004). Las altas concentraciones de materia orgánica en Xochimilco pueden favorecer la presencia de este hongo en el agua, como se observó en el lago Dianchi que está severamente contaminado.

Ophioceras commune es una especie dulceacuícola de amplia distribución. Crece sobre madera sumergida en Panamá, Hong Kong, China, Japón, Estados Unidos y México (Shearer et al., 1999; Tsui et al., 2001; Cai et al., 2002; González y Chavarria, 2005). En la temporada de lluvias se encuentra abundantemente en Xochimilco en bloques de madera de pino.

En la evaluación espacial se observan diferencias en los valores de la diversidad y abundancia de cada ascomicete entre las 4 estaciones de muestreo (Cuadro 1). Asimismo, los datos de la materia orgánica, el fósforo y el número de bacterias coliformes totales y fecales variaron entre los puntos de muestreo (Cuadro 4), lo cual puede deberse al diferente grado de urbanización y actividades diversas que se realizan en los márgenes del canal turístico Santa Cruz, que propician una gran heterogeneidad de hábitats, tal como sucede en todos los cuerpos de agua dulce de Xochimilco (Zambrano et al., 2009).

La tercera estación de muestreo del canal turístico Santa Cruz presentó la mayor diversidad y el mayor número de ascomicetes dulceacuícolas estrictos y también fue donde se registró la concentración más alta de oxígeno y la menor concentración de materia orgánica. Este resultado se debe posiblemente a que la tercera estación está situada en la zona de mayor importancia turística del canal, por lo que recibe un alto mantenimiento y vigilancia. En sus márgenes se desarrollan actividades de gran tradición cultural, como

Cuadro 4. Valores de algunos factores abióticos y bióticos del canal turístico Santa Cruz, Xochimilco, que se tomaron durante la realización de los muestreos

\begin{tabular}{|c|c|c|c|c|c|c|c|c|}
\hline & \multicolumn{4}{|c|}{ Temporada seca } & \multicolumn{4}{|c|}{ Temporada de lluvias } \\
\hline & \multicolumn{8}{|c|}{ Estaciones de muestreo } \\
\hline & 1 & 2 & 3 & 4 & 1 & 2 & 3 & 4 \\
\hline $\mathrm{MO} \mathrm{mg} / \mathrm{LO}_{2}$ & 154 & 251 & 88 & 151 & 555 & 295 & 25 & 75 \\
\hline Fósforo $\mathrm{mg} / \mathrm{L}$ & 5.8 & 5.6 & 7.6 & 6.7 & 23.0 & 17.1 & 16.0 & 14.2 \\
\hline $\mathrm{pH}$ & 6.2 & 7.2 & 6.7 & 7.6 & 8.0 & 7.0 & 7.0 & 7.0 \\
\hline $\mathrm{O}_{2} \mathrm{ml} / \mathrm{L}$ & 3.8 & 3.8 & 4.8 & 3.8 & 6.9 & 7.0 & 7.0 & 7.0 \\
\hline Temp ${ }^{\circ} \mathrm{C}$ & 21.0 & 20.0 & 22.0 & 23.0 & 21.5 & 19.8 & 21.0 & 19.8 \\
\hline Coliformes totales NMP/ml & 1600 & 2400 & 1600 & 52 & 83 & 2400 & 2400 & 54 \\
\hline Coliformes fecales NMP/ml & 900 & 42 & 12 & 16 & 58 & 920 & 83 & 29 \\
\hline Precipitación mm & & & 4.4 & & & & 5.8 & \\
\hline
\end{tabular}

$\mathrm{MO}=$ materia orgánica 
el cultivo y venta de diversas flores. En la primera estación se registró la diversidad más baja y la mayor abundancia de ascomicetes terrestres (Cuadro 1), también se registraron altos niveles de materia orgánica y bajas concentraciones de oxígeno (Cuadro 4), lo que probablemente favoreció la presencia de especies características de ambientes contaminados con materia orgánica, como Podospora sp. (Lou et al., 2004). Este resultado también coincide con el alto grado de urbanización, poca vigilancia y bajo mantenimiento que presenta esa región del canal (Fig. 2).

Los resultados de la evaluación temporal de la diversidad de los ascomicetes del canal turístico Santa Cruz muestran que en regiones templadas las comunidades de hongos dulceacuícolas se ven afectadas principalmente por las variaciones en la temperatura (Luo et al., 2004), mientras que en regiones tropicales y subtropicales la precipitación es el principal factor que afecta la composición de la comunidad (Tsui, 1999). En este estudio se observaron diferencias en la diversidad y en la abundancia de cada ascomicete en las 2 temporadas (Cuadro 2). A excepción de A. oligospora y A. aquatica, todos los hongos presentaron una disminución en su abundancia entre la temporada seca y la de lluvias. Lo anterior puede deberse al efecto de dilución producido por el volumen de agua que ingresa al canal como resultado de la precipitación registrada en la temporada de lluvias (Cuadro 4). En el lago de Pátzcuaro, Bernal-Brooks et al. (2003) observan que este efecto de dilución afectó el potencial de crecimiento de las algas debido a los cambios en las propiedades físicas y químicas del agua. En el caso de los ascomicetes dulceacuícolas del canal turístico Santa Cruz, este efecto de dilución disminuyó la probabilidad de que un número grande de esporas pudieran colonizar un sustrato. Zambrano et al. (2008) observaron diferencias entre diversos parámetros en varios canales de Xochimilco en diferentes años y temporadas y una alta heterogeneidad en los valores registrados. Estas diferencias entre los diversos parámetros pueden favorecer el desarrollo de algunas especies y limitar la presencia de otras. Ascolacicola aquatica fue la especie dulceacuícola que presentó la mayor abundancia en la temporada de lluvias, lo cual pudo deberse al aumento de restos vegetales que ingresaron al agua del canal por el efecto de la lluvia, favoreciendo el desarrollo de este organismo.

\section{Agradecimientos}

Al Posgrado en Ciencias Biológicas de la Universidad Nacional Autónoma de México, por el apoyo concedido durante la realización de este proyecto; al Consejo Nacional de Ciencia y Tecnología, por la beca doctoral otorgada; al Dr. Richard Hanlin, por sugerir la investigación de los ascomicetes acuáticos de Xochimilco y por revisar el Abstract; al Dr. Agustín Quiroz del Laboratorio de Vegetación Acuática del Instituto de Biología, UNAM, por su apoyo en la realización de los análisis de materia orgánica y fósforo de ortofosfatos; a la Dra. Concepción Toriello de la Facultad de Medicina de la Universidad Nacional Autónoma de México, por permitir la realización de los análisis bacteriológicos en el laboratorio a su cargo. Este trabajo fue financiado por el Consejo Nacional de Ciencia y Tecnología mediante el proyecto CONACyTSEP 60502, otorgado a MCG, Instituto de Biología, UNAM.

\section{Literatura citada}

American Public Health Association (APHA).1992. Standard methods for the examination of water and wastewater, $18^{\mathrm{a}}$ edición. Washington, D.C.

Au, D. W. T., I. J. Hodgkiss y Ln. L. P. Vrijmoed. 1992. Fungi and cellulolytic activity associated with decomposition of Bauhinia purpurea leaf litter in a polluted and unpolluted Hong Kong waterway. Canadian Journal of Botany 70:10711079.

Aveskamp, M. M., J. De Gruyter y P. W. Crous. 2008. Biology and recent development in the systematic of Phoma, a complex genus of major quarantine significance. Fungal Diversity 31:1-18.

Bernal-Brooks, F. W., L. Dávalos-Lind y T. D. Lind. 2003. Seasonal and spatial variations in algal growth potential and growth-limiting nutrients in a shallow endoreic lake: Lake Pátzcuaro (Mexico). Lake and Reservoirs Research and Management 8:83-93.

Bills, G. y J. Polishook, 1994. Abundance and diversity of microfungi in leaf litter of a lowland rain forest in Costa Rica. Mycologia 86:187-198.

Cai, L., C. K., M. Tsui, K. Zhang y K. D. Hyde, 2002. Aquatic fungi from Lake Fuxian, Yunnan, China. Fungal Diversity 9:57-70.

Cai, L., K. Q. Zang y K. D. Hyde. 2003. Freshwater ascomycetes. In Freshwater mycology, C. K. M. Tsui y K. D. Hyde (eds.). Fungal Diversity Research Series 10:275-326.

Cai, L., K. F. Ji y K. D. Hyde. 2006. Variations between freshwater and terrestrial fungal communities on decaying bamboo culms. Antonie van Leeuwnhoek Journal of Microbiology 89:293-301.

Carranco, P. D., A. Hernández y F. Rivera. 1984. Soil and aquatic fungi in a waste-stabilization pond system of the State of Mexico, Mexico. Water, Air and Soil Pollution 23:249-256.

Castañeda, R. F., G. P. Heredia, R. M. Arias, M. Saikawa, D. W. Minter, M. Stadler, J. Guarro y C. Decock. 2004. Two new hyphomycetes from rainforest of Mexico, and Briansuttonia, a new genus to accommodate Corynespora alternarioides. Mycotaxon 89:297-305. 
Castañeda, R. F., G. P. Heredia, R. M. Arias, M. Stadler y D. W. Minter. 2005. Two hyphomycetes from submerged material of Mexico. Mycotaxon 91:333-337.

Chavarria, A. 2003. Estudio preliminar de los hongos del canal Santa Cruz, laguna Xaltocán y canal Xaltocán, Delegación Xochimilco, Distrito Federal. Tesis, Facultad de Ciencias, UNAM, México, D.F. 69 p.

Chavarria, A. 2005. Diversidad de los ascomicetes y sus anamorfos del ambiente acuático de la Delegación Xochimilco, Ciudad de México. Tesis de maestría, Instituto de Biología, UNAM, México, D.F. 73 p.

Choi, Y. W., K. D. Hyde y W. H. Ho. 1999. Single spore isolation of fungi. Fungal Diversity 3:29-38.

CONAGUA (Comisión Nacional del Agua). 2009. http://www. conagua.gob.mx/

Domchs, K. H., W. Gams y T. H. Anderson. 1980. Compendium of soil fungi. Academic, New York. 859 p.

Fryar, S. C., J. Davies, W. Booth, I. J. Hodgkiss y K. D. Hyde. 2004. Succession of fungi on dead and live wood in brackish water Brunei. Mycologia 96:219-225.

Garnett, H., F. Bärlocher y D. Giberson. 2000. Aquatic hyphomycetes in Catamaran brook: colonization dynamics, seasonal patterns, and logging effects. Mycologia 92:29-41.

Goh, T. K. y C. K. M. Tsui. 2003. Key to common dematiaceous hyphomycetes from freshwater. In Freshwater mycology, C. K. M. Tsui y K. D. Hyde (eds.). Fungal Diversity Research Series 10:325-343.

Goh, T. K. y K. D. Hyde. 1996. Biodiversity of freshwater fungi. Journal of Industrial Microbiology 17:328-345.

González, M. C. y A. Chavarria. 2005. Some freshwater ascomycetes from Mexico. Mycotaxon 91:315-322.

Hawksworth, D. L. 2001. The magnitude of fungal diversity: 1.5 million species estimated revised. Mycological Research 105:1422-1432.

Heredia, G., J. Mena-Portales, A. Mercado-Sierra y M. Reyes. 1997. Tropical hyphomycetes of Mexico. II. Some species from the tropical Biology Station "Los Tuxtlas", Veracruz, Mexico. Mycotaxon 64:203-233.

Ho, W. W., K. D. Hyde y I. J. Hodgkiss. 1997. Ascomycetes from tropical freshwater habitats: the genus Savoriella, with two new species. Mycological Research 101:803-809.

Ho, W., H. Yanna, K. D. Hyde y I. J. Hodgkiss. 2002. Seasonal and sequential occurrence of fungi on wood submerged in Tai Po forest stream, Hong Kong. Fungal Diversity 10:2143.

Hyde, K. D. y T. K. Goh. 1998. Fungi on submerged wood in Lake Barrine, north Queensland, Australia. Mycological Research 102:739-749.

Hyde, K. D. 2001. Where are the missing fungi? Does Hong Kong have any answers? Mycological Research 105:15141518.

Hyde, K. D. y T. K. Goh. 2003. Adaptations for dispersal in filamentous freshwater fungi. In Freshwater mycology, C. K. M. Tsui y K. D. Hyde (eds.) Fungal Diversity Research Series 10:231-258.

Hyde, K. D., T. K. Goh. y T. D. Steinke. 1998. Fungi on submerged wood in the Palmiet River, Durban, South Africa. South African Journal of Botany 64:151-162.
Jones, E. B. G. 1971. Aquatic fungi. In Methods in microbiology, vol. 4, C. Booth (ed.). Academic, London. p. 335-365.

Juárez-Figueroa, L. A., J. Silvia-Sánchez, F. J. Uribe-Salas y E. Cifuentes-García. 2003. Microbiological indicators of water quality in the Xochimilco canals, Mexico City. Salud Pública de México 45:389-395.

Kane, D. F., W. Y. Tam y E. B. G. Jones. 2002. Fungi colonising and sporulating on submerged wood in the River Severn, UK. Fungal Diversity 10:45-55.

Krauss, G., F. Bärlocher y G. -J. Krauss. 2003. Effects of pollution on aquatic hyphomycetes. In Freshwater mycology, C. K. M. Tsui y K. D. Hyde (eds.) Fungal Diversity Research Series 10:212-230.

Kirk, P. M., P. F. Cannon, D. W. Minter y J. A. Stalpers. 2008. Dictionary of the fungi, décima edición. CAB International, Wallingford. $784 \mathrm{p}$.

Luo, J., J. Yin, L. Cai, K. Zhang y K. D. Hyde. 2004. Freshwater fungi in Lake Dianchi, a heavily polluted lake in Yunnan, China. Fungal Diversity 16:93-112.

Martínez, S. 2009. Diversidad de los ascomicetes microscópicos lignícolas del estanque de los manatíes de la Reserva Ecológica Yumká, Centro de Interpretación y Convivencia con la Naturaleza, Villahermosa, Tabasco. Tesis, División Académica de Ciencias Biológicas, Universidad Juárez Autónoma de Tabasco, Villahermosa. 96 p.

Moreno, C. 2001. Manual de métodos para medir la biodiversidad. Textos Universitarios, Universidad Veracruzana, Xalapa, Veracruz. $81 \mathrm{p}$.

Pascoal, C., L. Marvanova y F. Cássio. 2005. Aquatic hyphomycete diversity in streams of northwest Portugal. Fungal Diversity 19:109-128.

Pfister, D. H. y M. E. Liftik. 1995. Two Arthrobotrys anamorphs from Orbilia auricolor. Mycologia 87:684-688.

Révay, A. y J. Gönczöl. 1990. Longitudinal distribution and colonization patterns of wood-inhabiting fungi in a mountain stream in Hungary. Nova Hedwigia 51:505-520.

Rosique, E. 2008. Evaluación de la diversidad de los hongos microscópicos dulceacuícolas del estado de Tabasco, México. Tesis, doctorado Instituto de Biología. UNAM, México, D.F. 256 p.

Rosique, E., M. C. González, y J. Cifuentes. 2008. New records of three freshwater ascomycetes from an urban lagoon of Tabasco, Mexico. Mycotaxon 105:249-256.

Shearer, C. A. y J. L. Crane. 1986. Illinois fungi XII. Fungi and myxomycetes from wood and leaves submerged in southern Illinois swamps. Mycotaxon 25:527-538.

Shearer, C. A., J. L. Crane y W. Chen. 1999. Freshwater Ascomycetes: Ophioceras species. Mycologia 91:145-156.

Shearer, C. A., E. Descals, B. Kohlmeyer, J. Kohlmeyer, L. Marmanová, D. Padgett, D. Porter, H. A. Raja, P. J. Schmit, H. A. Thorton y H. Voglymayr. 2007. Fungal biodiversity in aquatic habitats. Biodiversity and Conservation 16:49-67.

Sivichai, S., E. B. G. Jones y N. L. Hywel-Jones. 2000. Fungal colonization of wood in a freshwater stream at Khao Yai National Park, Thailand. Fungal Diversity 5:71-88.

Sørensen, T. 1948. A method of stablishing groups of equal amplitude in plant sociology based on similarity of species content and its application to analyses of the vegetation on 
Danish commons. Kongelige Danske Videnskabernes Selskab Biologiske Skrifter 5:1-34.

Tsui, C. K. M. 1999. Biodiversity and longitudinal distribution of fungi on submerged wood, with reference to human disturbance. PhD Thesis University of Hong Kong. 307 p.

Tsui, C. K. M., K. D. Hyde y I. J. Hodgkiss. 2001. Longitudinal and temporal distribution of freshwater ascomycetes and dematiaceous hyphomycetes on submerged wood in the Lam Tsuen River, Hong Kong. Journal of North American Benthological Society 20:533-549.

Tsui, C. K. M., K. D. Hyde y I. J. Hodgkiss. 2003. Methods for investigating the biodiversity and distribution of freshwater ascomycetes and anamorphic fungi on submerged wood. In
Freshwater mycology, C. K. M. Tsui y K. D. Hyde (eds.). Fungal Diversity Research Series 10:195-209.

Vukojevic, J., D. Franic-Mihajlovic y S. Duletic-Lausevic. 1997. Soil micromycetes in the aquatic ecosystem of Vlasinsko Lake and its tributaries. Mycologia Helvetica 9:121-136.

Wong, M .K. M., T. K. Goh, I. J. Hodgkiss, K. D. Hyde, V. M. Ranghoo, C. K. M. Tsui, W. H. Ho, W. S. W. Wong y T. K. Yuen. 1998. Role of the fungi in freshwater ecosystems. Biodiversity and Conservation 7:1187-1206.

Zambrano, L., V. Contreras, M. Mazari-Hiriat y A. E. ZarcoArista. 2009. Spatial heterogeneity of water quality in a highly degraded tropical freshwater ecosystem. Environmental Management 43:249-263. 